\title{
Simultaneous measurement of antroduodenal motility, gastric emptying, and duodenogastric reflux in $\operatorname{man}^{1}$
}

\author{
W. D. W. REES ${ }^{2}$, V. L. W. GO, AND J.-R. MALAGELADA ${ }^{3}$ \\ From the Gastroenterology Unit, Mayo Clinic, Rochester, Minnesota, USA
}

SUMMARY In six healthy individuals, the relationship between antroduodenal motor activity, duodenogastric reflux, and gastric emptying were simultaneously examined by combined use of multiple marker perfusion and miniature strain gauge transducers. An interdigestive pattern of motor activity was observed during the fasting period; duodenogastric reflux was of variable magnitude, but reproducible in each individual. Fasting reflux was significantly reduced during phase III activity of the interdigestive complex. Administration of $0.15 \mathrm{M}$ sodium chloride into the stomach resulted in minor and inconsistent changes in antroduodenal motility, despite the rapid and similar pattern of gastric emptying in the six subjects. This study supports the concept that motor activity in the antroduodenal region does not affect gastric emptying of inert, isotonic fluids but may be involved in the regulation of duodenogastric reflux.

The relationship between antroduodenal motor activity, gastric emptying, and duodenogastric reflux remains controversial in man.

Recent studies in the dog after vagal denervation (Wibur and Kelly, 1973) and gastric resection (Dozois et al., 1971) suggest that antral motility regulates solid particle emptying and fundal tone modulates liquid phase emptying. Indirect evidence supporting this concept has also been gathered in man from studies of gastric emptying on postgastric surgery patients (Clarke et al., 1973; AlexanderWilliams et al., 1973; Howlett et al., 1976). Duodenal motility and pyloric sphincter 'tone' may also contribute by modifying the resistance to flow from the stomach (Weisbrodt et al., 1969; Fisher and Cohen, 1973), although others have not found the

\footnotetext{
'Supported in part by Grant AM 6908 from The National Institutes of Health, Bethesda, MD.

${ }^{2}$ Dr. Rees is the recipient of grants from The Medical Research Fund and the Gastrointestinal Foundation Trust, University Hospital of Wales, Wales, UK. Present address: Dr. W. D. W. Rees, Department of Medicine, University of Manchester, Hope Hospital, Eccles Old Road, Salford M6 8HD, England.

'Address for reprints: Dr. Juan-R. Malagelada, Gastroenterology Unit, Mayo Clinic, Rochester, MN 55901. Dr. Malagelada is the recipient of Academic Career Development Award No. 1 K04 AM 00330-01 from the National Institutes of Health.
}

Received for publication 13 June 1979 pylorus to behave as a true sphincter (Atkinson et al., 1957; Andersson and Grossman, 1965; Kaye et al., 1976).

Duodenogastric reflux has been documented in man, but its magnitude during the fasting state has been quite variable ranging from 8 to $20 \%$ (Go et al., 1970; Gustke et al., 1970). Intragastric administration of a saline bolus decreases the magnitude of reflux in man (Gustke et al., 1970), while increasing reflux in the dog (Kelly and Code, 1977). The precise mechanisms responsible for regulating are unknown but antroduodenal motor coordination may play an important role (Kelly and Code, 1977; Johnson et al., 1973).

Some of the current controversies are related to the difficulty in measuring gastric emptying, reflux, and antroduodenal motility simultaneously in man. The present study was designed to examine these three physiological events in an integrated fashion using a double marker duodenal perfusion technique for measuring gastric emptying and reflux and miniature strain gauge pressure transducers for measuring antroduodenal motor activity. We performed our observations both during fasting and after intragastric administration of an isotonic saline load. Isotonic saline causes only minimal stimulation of gastric secretion and its emptying is not delayed by duodenal osmoreceptors (Meeroff et al., 1975). 


\section{Methods}

The studies were performed on 12 healthy volunteers, aged 18-28 years, who had given informed consent. Six of the volunteers participated in the saline local tests and the remaining six in the validation studies.

\section{MATERIALS}

Four miniature strain gauge pressure transducers (Millar Instruments, Houston, Texas, Model PC 350) were attached $3.5 \mathrm{~cm}$ apart at $20,23.5,27$, and $30.5 \mathrm{~cm}$ from the tip of a triple lumen polyvinyl duodenal tube. One lumen was used to perfuse fluid into the duodenum opposite the ampulla of Vater, the second to aspirate fluid at the ligament of Treitz, and the third acted as an air vent to facilitate aspiration. A fingercot containing $3 / 4 \mathrm{ml}$ of mercury was attached to the distal end of the tube and facilitated its passage into the duodenum. The total diameter of the tube was $6 \mathrm{~mm}$.

\section{PROCEDURE}

After an overnight fast (12 hours), the duodenal tube was positioned fluoroscopically with the aspiration site at the ligament of Treitz, the perfusion site at the ampulla of Vater, and the four transducers straddled across the gastroduodenal junction, two being in the antrum, and two in the duodenum. A $14 \mathrm{~F}$ gastric sump tube was then positioned with its tip in the gastric antrum and the volunteers adopted a sitting posture for the remainder of the study (Fig. 1).

\section{EXPERIMENTAL DESIGN}

Validation studies: effect of gastroduodenal aspiration and perfusion on basal motor activity and pull-through studies

In six volunteers, one lumen of the polyvinyl tubewhich drained the duodenum at the level of the

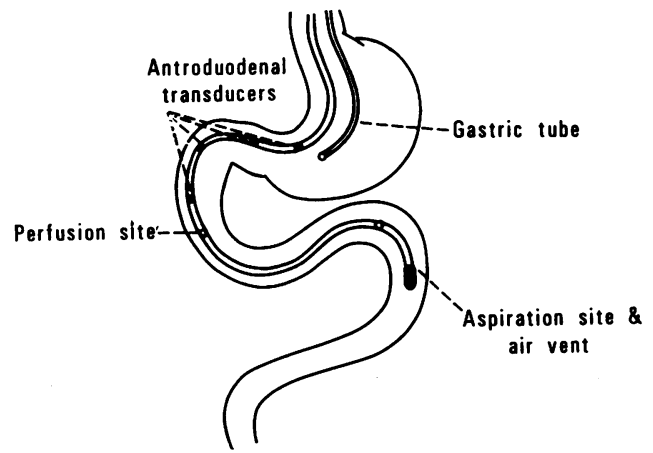

Fig. 1 Method used to simultaneously measure antroduodenal motor activity, gastric emptying, and duodenogastric reflux. ligament of Treitz - and the gastric sump tube were connected to suction apparatus, and the negative aspiration pressure varied from 0 to $-120 \mathrm{~mm} \mathrm{Hg}$ in increments of $40 \mathrm{~mm} \mathrm{Hg}$. Aspiration was then discontinued, and the second lumen of the intestinal tube-which opened opposite to the papilla of Vaterwas connected to a constant infusion pump. Fluid was introduced into the duodenum at rates of 0 to $8 \mathrm{ml} / \mathrm{min}$, in increments of $2 \mathrm{ml} / \mathrm{min}$, and the effect on motor activity was examined.

At the end of the study, during phase 1 or period of quiescence of the interdigestive motor cycle (Szurszewski, 1969; Code and Schlegel, 1973), the intestinal tube with attached transducers was slowly withdrawn at $1 \mathrm{~cm}$ intervals across the gastroduodenal and gastroesophageal junctions, and the pressure profiles of these two regions were examined.

Simultaneous maasurement of antroduodenal motility, gastric emptying, and duodenogastric reflux

In six volunteers, after intubation, duodenal perfusion with $0 \cdot 15 \mathrm{M}$ sodium chloride containing $50 \mu \mathrm{Ci} / 1$ of ${ }^{14} \mathrm{C}$-labelled polyethylene glycol was started at a rate of $2 \mathrm{ml} / \mathrm{min}$ and gastroduodenal contents were continually aspirated $(-40 \mathrm{~mm} \mathrm{Hg})$. One hour was allowed for the duodenal contents to reach equilibrium and the study was begun.

During a one hour fasting period, gastroduodenal contents were continually aspirated and pooled at 10 minute intervals. The volume of each sample was measured and an aliquot prepared for future analysis. Then a $400 \mathrm{ml} \mathrm{0.15} \mathrm{M}$ sodium chloride solution containing $50 \mu \mathrm{Ci}{ }^{3} \mathrm{H}$-labelled polyethylene glycol was instilled via the gastric tube over a 10 minute period, the duodenal contents being simultaneously aspirated and sampled as described. After sodium chloride administration and at the end of each 10 minute interval thereafter, a $10 \mathrm{ml}$ sample was aspirated from the stomach after thoroughly mixing the contents. At the end of one hour any remaining gastric content was completely aspirated and the stomach lavaged with $250 \mathrm{ml}$ solution of $0.15 \mathrm{M}$ sodium chloride, aliquots being prepared from both aspirate and lavage.

Each transducer was connected to a Gould four channel recorder (Gould Model 2400, Cleveland, Ohio) via a control unit (Millar Instruments, Model TC 100) that made possible zero and $100 \mathrm{~mm} \mathrm{Hg}$ calibration before intubation. The recording system employed DC amplifiers. The transducers were calibrated at room temperature (thermal stability of sensors $=1 \mathrm{~mm} \mathrm{Hg}$ over room temperature range $23^{\circ}$ to $38^{\circ} \mathrm{C}$ ). Motor activity was recorded from each of the four sites: proximal antrum, distal antrum, proximal duodenum, and mid-duodenum during the fasting and postsaline periods (Fig. 1). The sampling 
intervals were marked on each motility record to enable comparison of motor activity, rate of emptying and magnitude of reflux per 10 minutes.

\section{REPRODUCIBILITY STUDIES}

In five of the six subjects who took part in the saline load studies, a second identical study was carried out on a separate day in order to assess the reproducibility of the gastric emptying and reflux patterns.

\section{ANALYTICAL PROCEDURES AND}

MATHEMATICAL CALCULATIONS

Gastric emptying and duodenogastric reflux

${ }^{14} \mathrm{C}-\mathrm{PEG}$ and ${ }^{3} \mathrm{H}-\mathrm{PEG}$ concentrations in each gastric and duodenal aliquot were measured by liquid scintillation counting, and the rate of emptying of intragastric contents (saline and gastric secretion) was calculated using equations described in detail in a previous publication (Malagelada et al., 1976).

Fasting reflux was calculated from the volume aspirated from the stomach per 10 minutes and its ${ }^{14} \mathrm{C}-\mathrm{PEG}$ concentration (DPM/ml). Postsaline reflux was calculated using equations described by Malagelada et al., (1976) for calculating acid output per 10 minutes, the ${ }^{14} \mathrm{C}-\mathrm{PEG}$ concentration of each gastric aliquot being substituted for acid concentration. The 'output' of ${ }^{14} \mathrm{C}-\mathrm{PEG}$ obtained by this equation was equal to the amount refluxed into the stomach per 10 minutes. The amount of ${ }^{14} \mathrm{C}-\mathrm{PEG}$ refluxed per 10 minutes, during the fasting and post-saline periods, was then expressed as a percentage of the amount of ${ }^{14} \mathrm{C}-\mathrm{PEG}$ infused into the duodenum per 10 minutes. In order to validate these calculations, the amount of ${ }^{14} \mathrm{C}$-PEG present in the stomach at the end of the post-saline hour was calculated using the above equations and compared with that measured in the combined aspirate and lavage (Fig. 2). The close agreement between calculated and measured ${ }^{14} \mathrm{C}$-PEG content, for duplicate studies on five of the volunteers, reflects the accuracy and validity of the calculations.

\section{Antroduodenal motor activity}

Each motor record was divided into 10 minute periods corresponding to the sampling intervals. Motor activity at each of the four sites was calculated by measuring the area occupied by the pressure waves, the result being expressed as motility index in $\mathrm{mm}^{2} / 10 \mathrm{~min}$ for proximal antrum, distal antrum, proximal duodenum, and mid-duodenum.

\section{Results}

\section{VALIDATION STUDIES}

The effect of negative aspiration and duodenal perfusion on basal intraluminal pressure was examined during phase 1 of the interdigestive motor

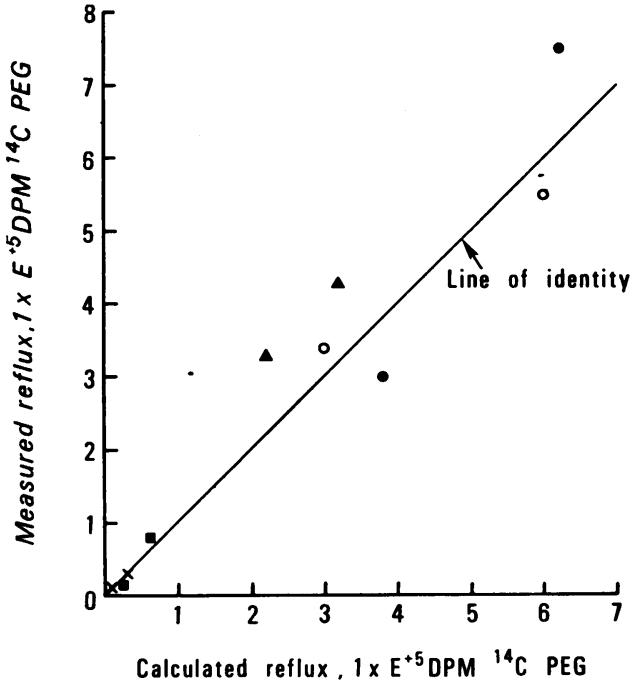

Fig. 2 Relationship between measured and calculated duodenal marker present in the stomach at the end of the post-saline hour in duplicate studies on five subjects (DPM - disintegrations per minute for $\left.{ }^{14} C-P E G\right)$. Each individual is identified by a different symbol.

complex. Increasing the negative aspiration pressure at the gastric antrum and distal duodenum from 0 to $-120 \mathrm{~mm} \mathrm{Hg}$ had no significant effect on resting intraluminal pressure at any of the four recording sites. Duodenal perfusion at rates varying from 0 to $8 \mathrm{ml} / \mathrm{min}$ similarly had no effect on recorded intraluminal pressure. Finally, the effect of manual aspiration of the gastric sump tube, using a 50-ml syringe, on motor activity was examined. Repeated aspiration and reinfusion of gastric contents had no effect on basal antral pressure.

Pull-through studies failed to reveal any tonic rise of basal pressure at the gastroduodenal junction in any of the six individuals studied. In contrast, a rise of the basal pressure was consistently detected at the lower oesophagus, the mean pressure \pm SE being $24 \pm 1 \mathrm{~mm} \mathrm{Hg}$ and the length of the tonic segment 2 to $3 \mathrm{~cm}$. A representative example of these pullthrough studies is shown in Fig. 3.

\section{FASTING ANTRODUODENAL MOTOR ACTIVITY} AND REFLUX

Fasting motor activity consisted of periods of quiescence (phase I) alternating with periods of activity; the latter consisting of random but persistent activity (phase II) followed by a short burst of intense motor activity that began simultaneously in the antrum and duodenum (phase III) (Szurszewski, 1969; Code and Schlegel, 1973). During phase III, antral and duodenal contractions occurred at 


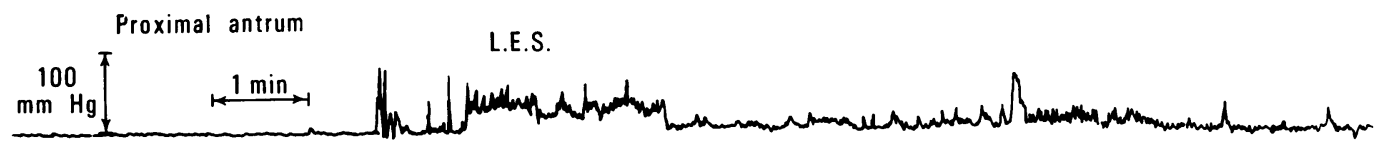

Distal antrum

L.E.S.

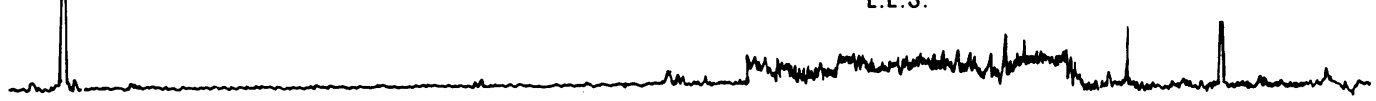

Proximal duodenum

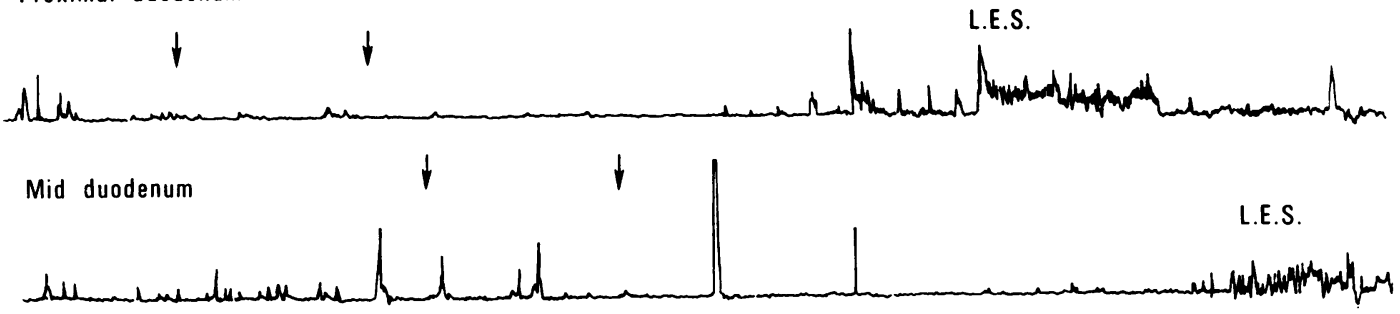

Fig. 3 Gastroduodenal and gastroesophageal pull-through tracing. LES lower esophageal sphincter; arrows indicate site of gastroduodenal junction.

frequencies of 3 and $11 /$ minute, respectively. A during the fasting hour, phases I and II only being representative example of interdigestive activity in one individual is shown in Fig. 4. An activity front (phasejIII) was observed in five out of six subjects present in the one subject (no. 2, Fig. 5).

The magnitude of fasting duodenogastric reflux varied among the six subjects, ranging from 0 to $80 \%$

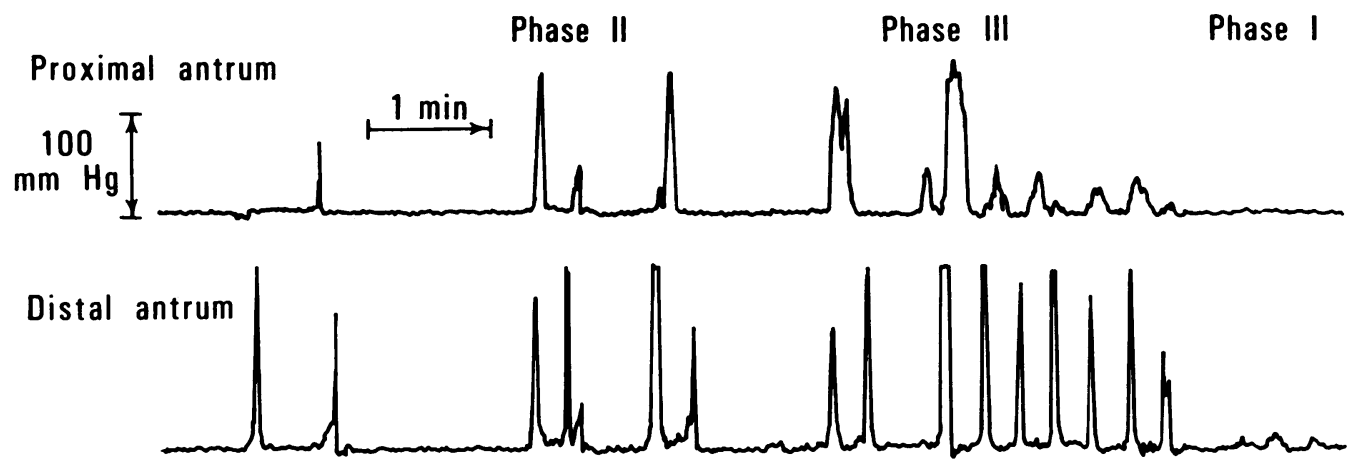

Proximal duodenum

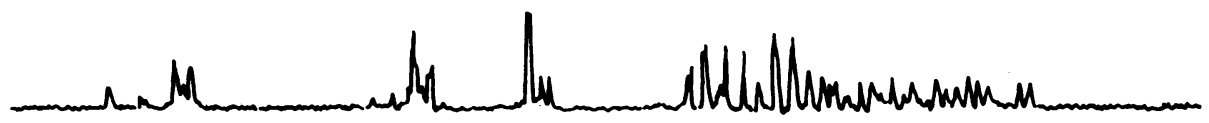

Mid duodenum

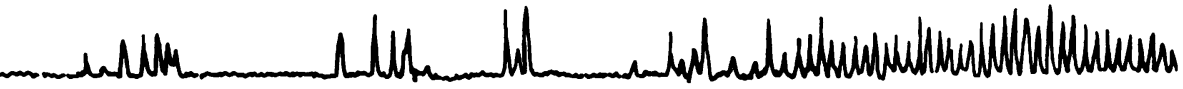

Fig. 4 Interdigestive motor activity recorded simultaneously from the antrum and duodenum. 


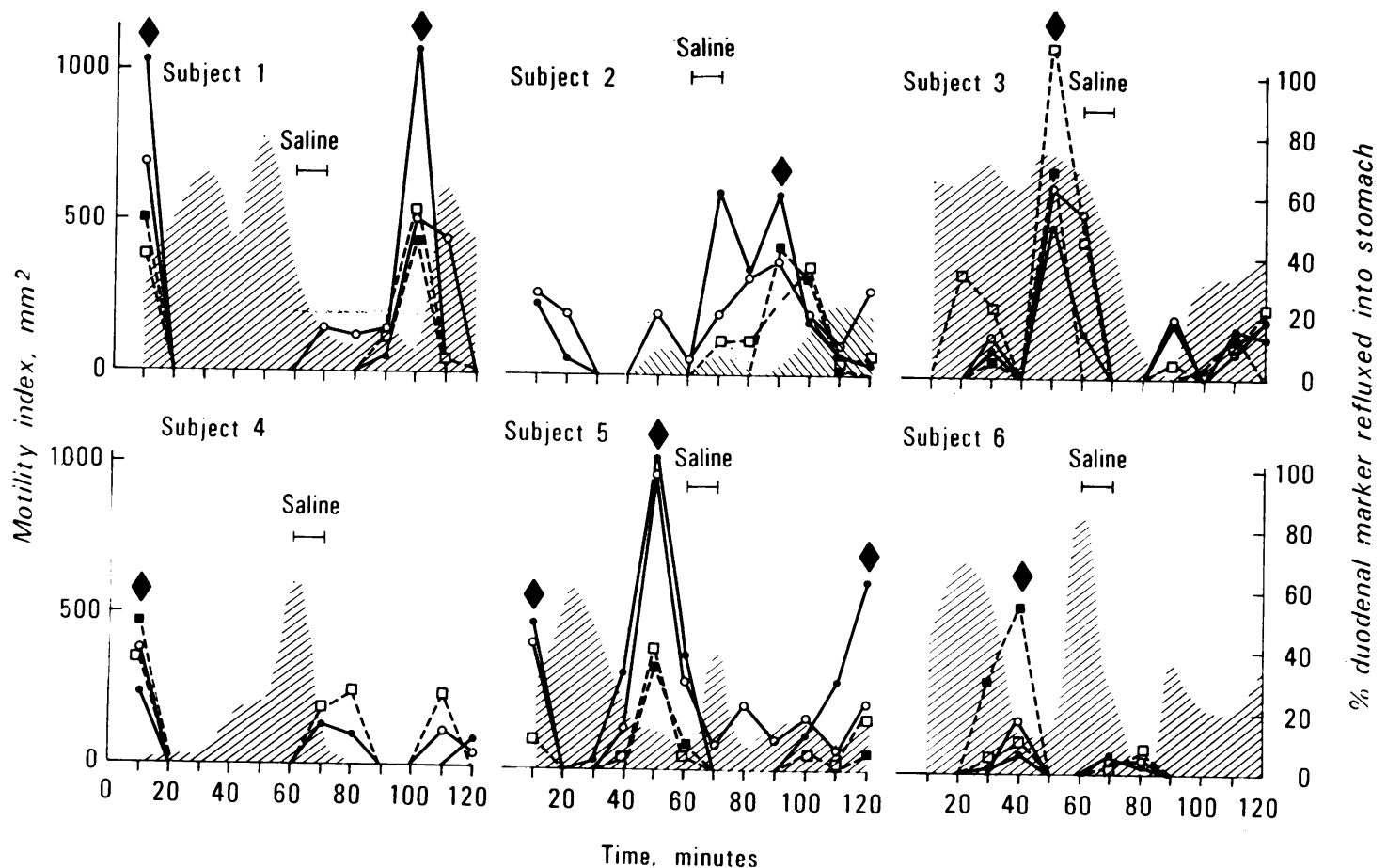

Fig. 5 Relationship between antrodudoenal motor activity and duodenogastric reflux in six subjects. Motility index in $\mathrm{mm}^{2}$ for: $\bigcirc-$, proximal antrum; $\bigcirc-$, distal antrum; $\square-$, proximal duodenum; and $\square-$, distal duodenum. $\checkmark$ phase III of the interdigestive complex. Cross-hatched area: percent of perfused duodenal marker refluxed into the stomach per 10 min.

in different individuals (Fig. 5). Analysis of the individual reflux patterns revealed that the magnitude of reflux was particularly low during phase III activity in subjects $1,4,5$, and 6 (Fig. 5). No phase III was present during the fasting period in subject 2 and the magnitude of reflux was not altered by phase III activity in subject 3 . The mean magnitude of reflux during phase III activity, in the five subjects, was significantly less than that during the remainder of the interdigestive cycle (mean $\pm \mathrm{SE}$ : phase III reflux $=25 \pm 14 \%$; phase I plus II reflux $=50 \pm 9 \%$, $\mathbf{P}<0.05$ by Student's $t$ test).

\section{POSTSALINE ANTRODUODENAL MOTOR}

ACTIVITY, REFLUX, AND GASTRIC EMPTYING Administration of sodium chloride $(400 \mathrm{ml})$, randomly during phase I of the interdigestive cycle, had no consistent effect on the pattern of antoduodenal motility (Fig. 6). In subject 1 , there was a slight increase in distal duodenal activity and an activity front appeared 40 minutes after instillation. In subject 2 , distal antral and proximal duodenal activity was increased and an activity front occurred 30 minutes after administration of saline. Phase I motor activity appeared unchanged in subjects 3 and 5 , an activity front appearing 60 minutes after instillation in subject 5 . In subjects 4 and 6 , there was a slight increase in duodenal and duodenal plus distal motor activity, respectively. Analysing the motility recordings for frequency, mean amplitude, and mean duration of contractions produced similar results as with the motility index (data not shown).

The rate of emptying of the saline solution was rapid, the mean $T_{1}^{1}$ for the six subjects being 17 minutes (range: 12 to 24 minutes). Despite the inconsistent antroduodenal motor response to the saline solution, the pattern of emptying was remarkably similar in the six subjects (Fig. 5) and there was no relationship between the rate of emptying and motor activity at any of the four transducer sites.

The percentage of duodenal marker refluxed was reduced after saline administration in five out of the six subjects (Fig. 5), the overall mean reflux for the six subjects being significantly less than fasting reflux at 20,30, and 40 minutes postsaline (overall mean \pm SE: fasting $=35 \pm 10 \%$; postsaline, $20 \mathrm{~min}=4 \pm 2 \%$; $30 \mathrm{~min}=12 \pm 7 \% ; 40 \mathrm{~min}=14 \pm 6 \% ; \mathrm{P}<0.05)$. After 


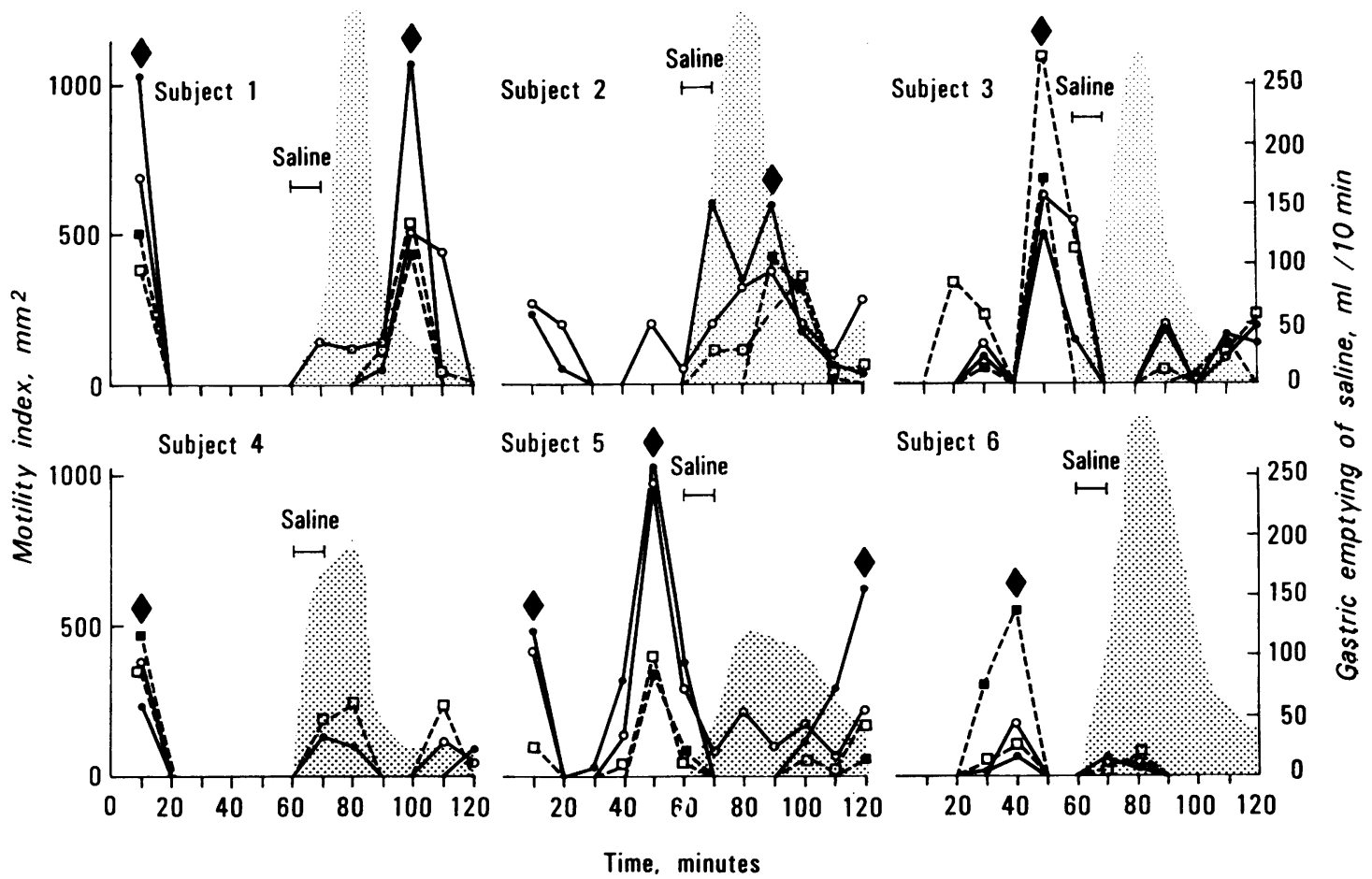

Fig. 6 Relationship between antroduodenal motor activity and gastric emptying of a saline meal in six subjects. Motility index in $\mathrm{mm}^{2}$ for: $\bigcirc-$, proximal antrum; --- , distal antrum; $\square-$, proximal duodenum; and $\square-$, distal duodenal. phase III of the interdigestive complex. Stippled area: gastric emptying of saline in ml per $10 \mathrm{~min}$.

40 minutes the post-saline reflux was not significantly different from fasting reflux.

\section{REPRODUCIBILITY OF EMPTYING AND \\ REFLUX PATTERNS}

The results of duplicate studies on five of the six subjects are illustrated in Fig. 7. The rate and pattern of gastric emptying of saline and the magnitude of fasting duodenogastric reflux and its response to saline administration was similar in the first and second studies, thus confirming the reproducibility of the results obtained by the above technique.

\section{Discussion}

The results of this study show that administration of a $400 \mathrm{ml}$ sodium chloride bolus into the stomach during the quiescent period (phase I) of the interdigestive cycle had no consistent effect on antroduodenal motor activity. However, despite the inconsistent motor response, the sodium chloride solution emptied rapidly and with a similar pattern in all subjects. Our results tend to support the conclusions of Wilbur and Kelly (1973) and Dozois et al. (1971) that the antrum does not play a major role in regulating the emptying of liquids from the stomach.

The magnitude of duodenogastric reflux was assessed from the quantity of duodenal marker that entered the stomach. Two factors appeared to reduce reflux: firstly, gastric emptying of the sodium chloride solution and, secondly, the presence of gastroduodenal phase III motor activity. The effect of emptying on reflux could simply reflect the rapid flow of saline from stomach to duodenum, acting to dilute and 'wash' the infused duodenal marker distally. However, the effect of phase III activity of reflux suggests that antroduodenal motor activity does play a role in regulating regurgitation of duodenal contents into the stomach.

Some technical aspects of the study deserve further consideration. The method allows simultaneous, quantitative measurement of gastric emptying reflux, and motor activity in man by the use of a previously validated, intestinal perfusion technique (Malagelada et al., 1976) and intraluminal strain gauge pressure transducers. The accuracy of the reflux calculations was confirmed by the close relationship observed between the measured and calculated quantity of duodenal marker present in 

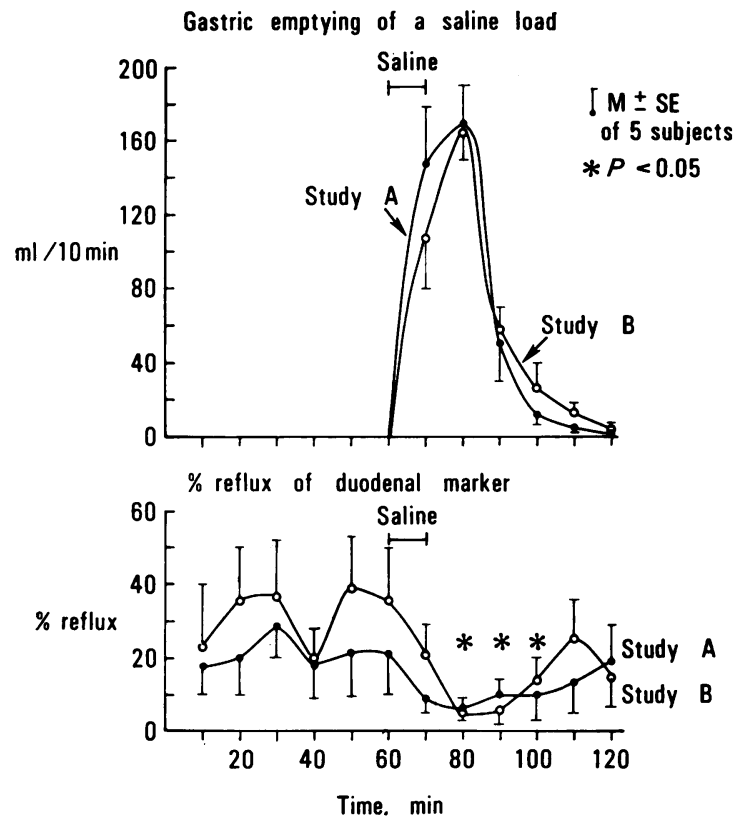

Fig. 7 Reproducibility of gastric emptying and duodenogastric reflux in five subjects. Asterisk represents a significant reduction in duodenogastric reflux after saline administration in both series of experiments.

the stomach at the end of the study. Although the gastric sampling techniques were different during the fasting and postsaline periods (continuous and intermittent, respectively), this is unlikely to account for the decrease in reflux for two reasons: first, Gustke et al. (1970) have previously shown that continuous versus intermittent gastric sampling has no effect on duodenogastric reflux in man, and, secondly, the magnitude of reflux during the late postsaline period was not significantly different from fasting reflux.

Intraluminal strain gauge transducers, used in the present study, have some advantages over the conventional perfused-catheter manometric technique. Transducers have optimal compliance and operate independently of a perfusion system. We could therefore use them to measure intraluminal pressure in conjunction with quantifying fluid flow without the need for mathematical correction for the volume of perfused fluid. At the same time, we showed by our validation studies that gastroduodenal aspiration and perfusion had no effect on basal intraluminal pressure.

The physiological significance of our study is that phasic pressure rises at the antroduodenal region play little or no role in regulating the emptying of inert fluids from the stomach. This finding is con- sistent with the concept, developed primarily in experimental animals, that emptying of such fluids is primarily a function of the fundic region (Dozois et al., 1971; Wilbur and Kelly, 1973). Our studies also suggest that phase III of the interdigestive motor cycle may play a part in reducing duodenogastric reflux. The extent to which the presence of a transpyloric tube might have influenced our results is difficult to estimate. We have earlier shown that duodenal intubation has no effect on gastric secretion or emptying after a mixed meal (Longstreth et al., 1975). Meeroff et al. (1973) found no differences in the rate of gastric emptying of normal saline measured by duodenal marker perfusion and published data based on gastric aspiration (George, 1968). With regard to measurements of intraluminal pressure, the question is unanswerable at the present time, as manometric recordings in man cannot be obtained without intubation. The present results, however, should stimulate further studies on the way in which motor activity in the upper gastrointestinal tract determines movement of luminal contents.

The authors thank Ms. Brenda Marben, Mrs. Judith A. Duens, Mr. Larry Stokes, and Mr. Richard Tucker for their technical help and Ms. Gaurdis Grube for her secretarial assistance.

\section{References}

Alexander-Williams, J., Donovan, I. A., Gunn, I. F., Brown, A., and Harding, L. K. (1973). The effect of vagotomy on gastric emptying. Proceedings of the Royal Society of Medicine, 66, 1102-1103.

Andersson, S., and Grossman, M. I. (1965). Profile of pH, pressure and potential difference at gastroduodenal junction in man. Gastroenterology, 49, 364-371.

Atkinson, M., Edwards, D. A., Honour, A. J., and Rowlands, E. M. (1957). Comparison of cardiac and pyloric sphincters: a manometric study. Lancet, 273, 918-922.

Clarke, R. J., and Alexander-Williams, J. (1973). The effect of preserving antral innervation and of a pyloroplasty on gastric emptying after vagotomy in man. Gut, 14, 300-307.

Code, C. F., and Schlegel, J. F. (1973). The gastrointestinal interdigestive housekeeper: motor correlates of the interdigestive myoelectric complex of the dog. Proceedings of the Fourth International Symposium on Gastrointestinal Motility, pp. 631-634. Mitchell Press: Vancouver.

Dozois, R. R., Kelly, K. A., and Code, C. F. (1971). Effect of distal antrectomy on gastric emptying of liquids and solids. Gastroenterology, 61, 675-681.

Fisher, R., and Cohen, R. (1973). Physiological characteristics of the human pyloric sphincter. Gastroenterology, 64, 67-75.

Go, V. L. W., Hofmann, A. F., and Summerskill, W. H. J. 
(1970). Simultaneous measurements of total pancreatic, biliary and gastric outputs in man using a perfusion technique. Gastroenterology, 58, 321-328.

Gustke, R. F., Varma, R. R., and Soergel, K. H. (1970). Gastric reflux during perfusion of the proximal small bowel. Gastroenterology, 59, 890-895.

Howlett, P. J., Sheiner, H. J., Barber, D. C., Ward, A. S., Perez-Avila, C. A., and Duthie, H. L. (1976). Gastric emptying in control subjects and patients with duodenal ulcer before and after vagotomy. Gut, 17, 542-550.

Hunt, J. N. (1963). The duodenal regulation of gastric emptying. Gastroenterology, 45, 149-156.

Johnson, A. G., Kirk, C. J. C., and March, C. S. (1973). Does pyloric competence depend on antro-duodenal coordination? Proceedings of the Fourth International Symposium on Gastrointestinal Motility, pp. 505-513. Mitchell Press: Vancouver.

Kaye, M. D., Mehta, S. J., and Showalter, J. P. (1976). Manometric studies of the human pylorus. Gastroenterology, 70, 477-480.

Kelly, K. A., and Code, C. F. (1977). Duodenal-gastric reflux and slowed gastric emptying by electrical pacing of the canine duodenal pacesetter potential. Gastroenterology, 72, 429-433.
Longstreth, G. F., Malagelada, J.-R., and Go, V. L. W. (1975). The gastric response to a transpyloric duodenal tube. Gut, 16, 777-780.

Malagelada, J.-R., Longstreth, G. F., Summerskill, W. H. J., and Go, V. L. W. (1976). Measurement of gastric functions during digestion of ordinary solid meals in man. Gastroenterology, 70, 203-210.

Meeroff, J. C., Go, V. L. W., and Phillips, S. F. (1973). Gastric emptying of liquids in man-quantification by duodenal recovery marker. Mayo Clinic Proceedings, 48, 728-732.

Meeroff, J. C., Go, V. L. W., and Phillips, S. F. (1975). Control of gastric empyting by osmolality of duodenal contents in man. Gastroenterology, 68, 1144-1151.

Szurszewski, J. H. (1969). A migrating electric complex of the canine small intestine. American Journal of Physiology, 217, 1757-1763.

Weisbrodt, N. W., Wiley, J. N., Overholt, B. F., and Bass, P. (1969). A relation between gastroduodenal muscle contractions and gastric emptying. Gut, 10, 543-548.

Wilbur, B. G., and Kelly, K. A. (1973). Effect of proximal gastric, complete gastric, and truncal vagotomy on canine gastric electric activity, motility and emptying. Annals of Surgery, 178, 295-303. 